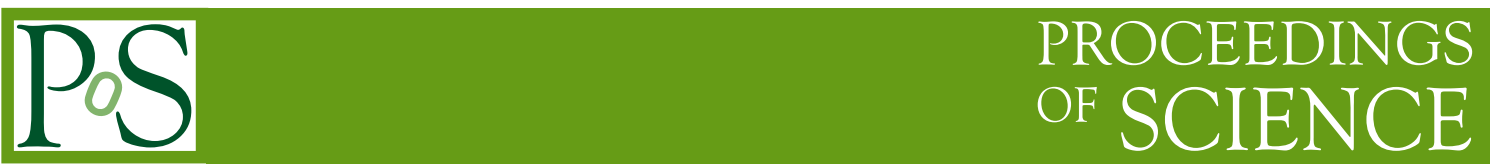

\title{
Hadrons with $c-s$ content: past, present and future
}

\author{
Elisabetta Prencipe* on behalf of the $\overline{\mathbf{P}}$ ANDA Collaboration \\ Jülich Forschungszentrum \\ E-mail: e.prencipe@fz-juelich.de
}

\begin{abstract}
The PANDA detector at FAIR aims to conduct an antiproton-proton experiment with a very high rate capability. It is expected to feature high mass resolution, more than 20 times better than achieved at B-factories. $\overline{\mathrm{P}} \mathrm{ANDA}$ is in a unique position to perform highly resolved mass scan, and to measure the width of very narrow charm and charmonium-like states, whose nature is still unknown, 12 years after their discovery. In this report, we present a method to determine the width of the $D_{s 0}^{*}(2317)^{+}$. We discuss the future perspectives of $\bar{P}$ ANDA, based on our present simulations, in relation with the recent measurements performed by $\mathrm{LHCb}$ and the performances in this field at the B factories.
\end{abstract}

53rd International Winter Meeting on Nuclear Physics, 26-30 January 2015

Bormio, Italy

\footnotetext{
* Speaker.
} 


\section{Introduction}

The sector of Charm and Charmonium physics is richer as expected according to the potential model predictions. New resonant states with quite unusual properties have been observed. Prominent examples are the $X(3872)$ and the charged $Z_{c}^{ \pm}(3900)$ in the Charmonium sector, and the $D_{s}$ mesons below the $D K$ threshold in the open-charm sector.

Strangeness, in combination with open and hidden Charm, is a topic still to be exploited, in both Charm and Charmonium field. Composite systems of heavy-light quarks have gained the attention of the Charm community since the discovery of the $D_{s 0}^{*}(2317)^{+}$, that was surprisingly found more than $100 \mathrm{MeV} / \mathrm{c}^{2}$ below the potential model predictions. As a consequence, new theoretical interpretations have been proposed to explain this resonant state, like hadro-charmonia, hybrids, tetraquarks and hadronic molecules. High quality calculations and measurements of the properties of these states are compulsory to decide among the various scenarios, and conclude on their nature.

We report here on a method to determine the width of the $D_{s 0}^{*}(2317)^{+}$with the PANDA experiment, proposing a tight mass scan in $100 \mathrm{keV} / \mathrm{c}^{2}$ steps.

\section{Motivation}

Potential models give reliable predictions of $D$ and $D_{s}$ masses [1,2]. These particles are bound systems of a heavy quark $(c)$, and a light quark $(u, d$ or $s)$. The $s$-quark represents a "transition" case between the sector of light quarks (e.g. $u$ and $d$ ), and heavy quarks (e.g., $c, b$, or $t$ ). Bound systems composed by a $c$-quark and a $s$-quark, e.g. the so-called $D_{s}$ mesons, are charged states. On the other hand, $D$ mesons can be neutral and charged.

The $D_{s 0}^{*}(2317)^{+}$was observed by BaBar in 2003 [3], at a mass value more than $100 \mathrm{MeV} / \mathrm{c}^{2}$ below the potential model predictions, and it was observed only in the decay to $D_{s}^{+} \pi^{0}$. To make the picture more complicated, the $D_{s 1}(2460)^{+}$was observed in 2004 [4]. Also in this case, the presence of a spin $1^{+}$state was predicted, but its mass was found $80 \mathrm{MeV}$ below the potential model prediction. This is not understood, because the $s$-quark could be considered as light up to a certain extent, and the $c$-quark is massive enough, so the perturbative calculations are expected to deliver reliable predictions. Therefore, several alternative theoretical interpretations have been proposed to explain this, and other $D_{s}^{(*)}$ resonant states. The interpretation of the $D_{s 0}^{*}(2317)^{+}$and the $D_{s 1}(2460)^{+}$as hadro-charmonia [5], hybrids [6], or pure $c \bar{s}$ states [7] have been suggested.

The $D_{s 0}^{*}(2317)^{+}$width is nowadays unknown: an upper limit of $3.8 \mathrm{MeV}$ has been set [8] to the width measurement of this very narrow state by experiments at B factories. The same stands for the $D_{s 1}(2460)^{+}$. In the recent theoretical work of Ref. [9], the $D_{s 0}^{*}(2317)^{+}$width is evaluated under the hypothesis that it is a pure $c \bar{s}$ state, and under the hypothesis of being a molecular state. The former case leads to a width of $\sim 30 \mathrm{keV}$, whereas in the latter case the width is predicted to be $\sim 133 \mathrm{keV}$. In this theoretical paper, there are arguments in favor of the molecular nature of the $D_{s 0}^{*}(2317)^{+}$. The two hypotheses differ basically because of the contribution of the $D^{+} K^{0}$ and $D^{0} K^{+}$loops to the rate of the process, which gives rise to an additional contribution of the hadronic width of the $D_{s 0}^{*}(2317)^{+}$of about $100 \mathrm{keV}$. To unambiguously conclude on the preferred molecular 
description of the state, a new generation of experiments able to scan the mass of the resonant state in $\sim 100 \mathrm{keV} / \mathrm{c}^{2}$ steps is needed.

In a $\bar{p} p$ process, it is possible to reach such a high mass resolution only with a very high beam momentum resolution. In this situation, the $D_{s}$ mesons can be produced as charged pairs. So, the process to be investigated could be $\bar{p} p \rightarrow D_{s}^{-} D_{s 0}^{*}(2317)^{+}$, where $D_{s}^{-}$is the ground state of the $c \bar{s}$ spectrum. In Fig. 1, the $c \bar{s}$ spectrum is reported, populated by many states, mainly discovered in the past 12 years. The minimum antiproton beam momentum that one needs to run, in order to produce the $D_{s}^{-} D_{s 0}^{*}(2317)^{+}$pair via $\bar{p} p$ annihilation, is $p_{\text {beam }}=8.80235 \mathrm{GeV} / \mathrm{c}$. Here, a mass resolution of $100 \mathrm{keV} / \mathrm{c}^{2}$ can be reached only if the beam momentum resolution is $\sim 10^{-5}$. An experiment with these features does not exist, presently. However, the future $\bar{P}$ ANDA experiment [10] will reach a beam momentum resolution $\Delta p / p$ of the order of $10^{-5}$ (in the high resolution mode), which is ideally what is needed to perform the challenging measurement of the width of the $D_{s 0}^{*}(2317)^{+}$ and the $D_{s 1}(2460)^{+}$. A crucial point is of course the amount of luminosity that is needed for such

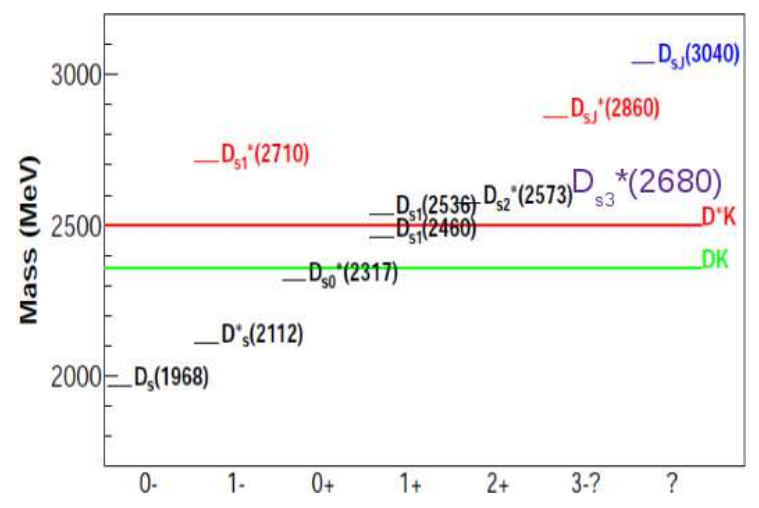

Figure 1: $c \bar{s}$ mass spectrum as function of the spin $\mathrm{J}^{P}$, today. The $D_{s}(2860)$ has been recently interpreted as an admixture of spin 1 and spin 3 states by LHCb [23].

a measurement. This number depends on the cross section of the $\bar{p} p \rightarrow D_{s}^{-} D_{S J}^{*+}$ process, which is still unknown: $\mathscr{L} \cdot \sigma \cdot \varepsilon=N$, where $\mathscr{L}$ is the integrated luminosity, $\sigma$ is the cross section of the process, $\varepsilon$ is the reconstruction efficiency for the resonant state, and $N$ is the number of fitted $D_{s}^{-} D_{s J}^{*+}$ pairs.

Several theoretical studies have been performed in the past to estimate the cross section of $\bar{p} p$ to open-charm. They have exploited different theoretical approaches (perturbative QCD [11, 12], baryon exchange [13] or quark model [14], (un)quenched lattice QCD calculations), leading to different conclusions.

In Fig. 2, the resultant cross section is estimated to be in the range $[1 ; 10]$ nb (quark model), and $[20 ; 30] \mathrm{nb}$ (baryon exchange model), as shown, respectively. The results of these models are based on $\mathrm{SU}(4)$ symmetry, which are expected to be only valid for $D\left(D_{s}\right)$ ground states [13]. For excited $D$ or $D_{s}$ states, the situation is more complicated. One cannot rely on predictions on the coupling constant evolution depending on the new energy scale. Perturbative calculations do not 
work in this case. One would need to look into a (semi)inclusive process, in which the charm quark has to hadronize into $D_{s 0}^{*}(2317)^{+}$. Not much is known so far about the fragmentation functions for this state.

In the most conservative quark model approach, the $D_{s 0}^{*}(2317)^{+}$, the $D_{s 1}(2460)^{+}$and the $D_{s}(2860)^{+}$are the only anomalies, in the $c \bar{s}$ spectrum. It is interesting to note that the $D_{s}(2860)^{+}$ is observed above the $D^{*} K$ threshold, with a larger width compared to that of the very narrow $D_{s 0}^{*}(2317)^{+}$and $D_{s 1}(2460)^{+}$, observed below the $D^{*} K$ threshold. Lattice QCD calculations (for example, as reported in Refs. $[15,16])$ suggest that $D K$ scattering amplitudes are required to obtain the large mass shifts of $180 \mathrm{MeV} / \mathrm{c}^{2}$ for the $D_{s 0}^{*}(2317)^{+}$. A study of the $B_{s}$ counterpart of these $D_{s}$ states would help to understand their nature. However, $B_{s}$ decays will be not part of the $\overline{\mathrm{P} A N D A}$ physics program, because the energy in the center of mass system that PANDA can reach is limited to $5.5 \mathrm{GeV} / \mathrm{c}^{2}$.

Regge trajectories are also used in the cross section calculations, for example in the process of $\bar{p} p$ to baryons. However, when developing calculations up to higher orders, divergences occur that are difficult to cure. Therefore, it is not easy to perform theoretical calculations in a rigorous way for the process $\bar{p} p \rightarrow$ open-charm, especially when excited states are involved. Data are needed, and an experiment providing a high-momentum resolution antiproton beam is crucial to perform these studies.

\section{The $\bar{P} A N D A$ approach}

A (semi)inclusive analysis for the channel $\bar{p} p \rightarrow D_{s}^{-} D_{s 0}^{*}(2317)^{+}$is proposed. It gives a larger cross section compared to the exclusive analysis. In the absence of a rigorous prediction for the cross section of $\bar{p} p \rightarrow D_{s}^{-} D_{s 0}^{*}(2317)^{+}$, we may consider valid for the $\bar{p} p \rightarrow D_{s}^{-} D_{s 0}^{*}(2317)^{+}$process, the same cross section prediction as for $\bar{p} p \rightarrow D_{s}^{-} D_{s}^{+}$(see Fig. 2). The calculation reported in Ref. [13] extends up to $p=8.5 \mathrm{GeV} / \mathrm{c}$, and the antiproton beam momentum, which is needed for this analysis, is $p_{\text {beam }} \geq 8.8 \mathrm{GeV} / \mathrm{c}$. We thus extrapolate our results under the assumption that the cross section of the $\bar{p} p \rightarrow D_{s}^{-} D_{s 0}^{*}(2317)^{+}$process is in the range [20;30] nb.

\subsection{Experimental overview}

Figure 3 shows the published results from the BaBar experiment [3, 4]. Clear peaks have been observed, corresponding to the $D_{s 0}^{*}(2317)^{+}$and the $D_{s 1}(2460)^{+}$, respectively. A structure in the $D_{s}^{+} \pi^{0}$ invariant mass, corresponding to the $D_{s 0}^{*}(2317)^{+}$, is supposed to have $J^{P}=0^{+}$. The search for other decays modes led to the discovery of an additional state, the $D_{s 1}(2460)^{+}$. This new state was seen by analyzing the invariant mass of $D_{s}^{+} \gamma$ and $D_{s}^{*+} \pi^{0}$. Since the $D_{s 1}(2460)^{+}$was not observed decaying to $D_{s}^{+} \pi^{0}$, we can conclude that it is not a spin- 0 resonance, but it has spin 1 . The angular analysis performed by BaBar favors this spin assignment.

If $D_{s 0}^{*}(2317)^{+}$and $D_{s 1}(2460)^{+}$are the $J^{P}=0^{+}$and $J^{P}=1^{+}$states, respectively, belonging to the same family in the $c \bar{s}$ spectrum, they are expected to decay to the channels summarized in Table 1. However, some of the decays are not observed. This disfavors the interpretation of the $D_{s 0}^{*}(2317)^{+}$and the $D_{s 1}(2460)^{+}$as the missing $J^{P}=0^{+}$and $J^{P}=1^{+}$states of the $c \bar{s}$ spectrum. In order to understand what these states are, more searches were performed. Their present status is summarized in Fig. 1, where many excited $D_{s}$ states are shown, even above the $D K$ threshold. 


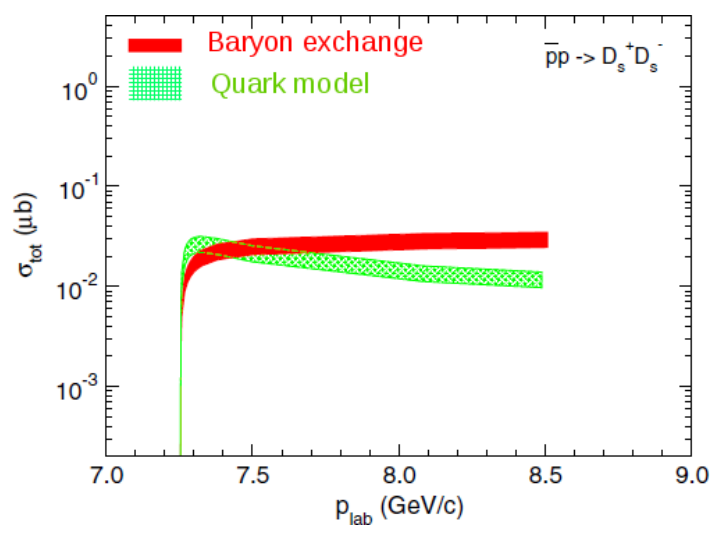

Figure 2: Cross section calculation of the $\bar{p} p \rightarrow D_{s}^{+} D_{s}^{-}$reaction within the SU(4) symmetry. Results are taken from Ref. [13].

Table 1: Summary of decay channels for the $D_{s 0}^{*}(2317)^{+}$and the $D_{s 1}(2460)^{+}$.

\begin{tabular}{lrc}
\hline \hline Decay channel & $D_{s 0}^{*}(2317)^{+}$ & $D_{s 1}(2460)^{+}$ \\
\hline$D_{s}^{+} \pi^{0}$ & Seen & Forbidden \\
$D_{s}^{+} \gamma$ & Forbidden & Seen \\
$D_{s}^{+} \pi^{0} \gamma$ non resonant & Allowed & Allowed \\
$D_{s}^{*}(2112)^{+} \pi^{0}$ & Forbidden & Seen \\
$D_{s 0}^{*}(2317)^{+} \gamma$ & - & Seen \\
$D_{s}^{+} \pi^{0} \pi^{0}$ & Forbidden & Allowed \\
$D_{s}^{+} \gamma \gamma($ non resonant $)$ & Allowed & Allowed \\
$D_{s}^{*}(2112)^{+} \gamma$ & Allowed & Allowed \\
$D_{s}^{+} \pi^{+} \pi^{-}$ & Forbidden & Seen \\
\hline \hline
\end{tabular}

From past experiments, we know that a good technique for measuring the width of a narrow state is to scan its mass. With this technique, and with a beam momentum resolution of $\sim 500 \mathrm{keV} / \mathrm{c}$, the experiment E760, for instance, measured the width of the $J / \psi: \Gamma=(99 \pm 12 \pm 6) \mathrm{keV}$ [17]. For comparison, $\overline{\mathrm{P}} \mathrm{PNDA}$ is designed to have a beam momentum resolution $\leq 100 \mathrm{keV} / \mathrm{c}$.

$\overline{\mathrm{P}}$ ANDA has multiple interests in analyzing the $\bar{p} p \rightarrow D_{s}^{-} D_{s 0}^{*}(2317)^{+}$channel, namely:

- a fit to the excitation function of the cross section, to extract the width $(\Gamma)$ of the $D_{s 0}^{*}(2317)^{+}$ and $D_{s 1}(2460)^{+}$;

- the production cross section determination; 


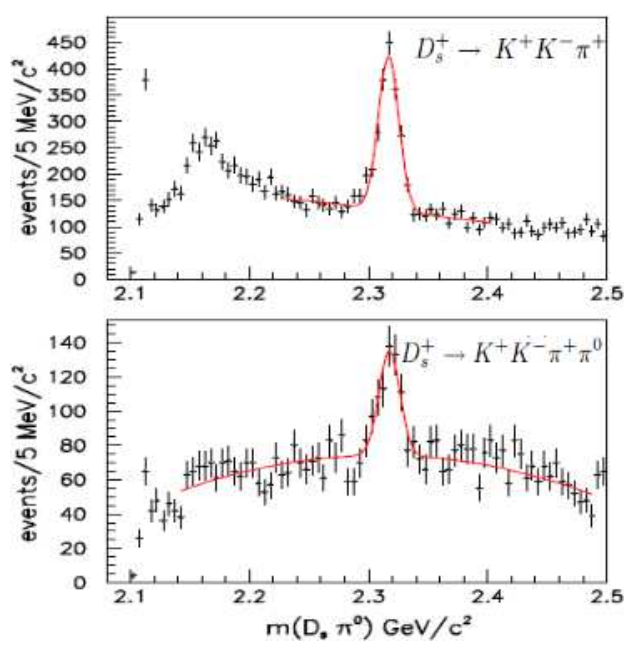

(a)

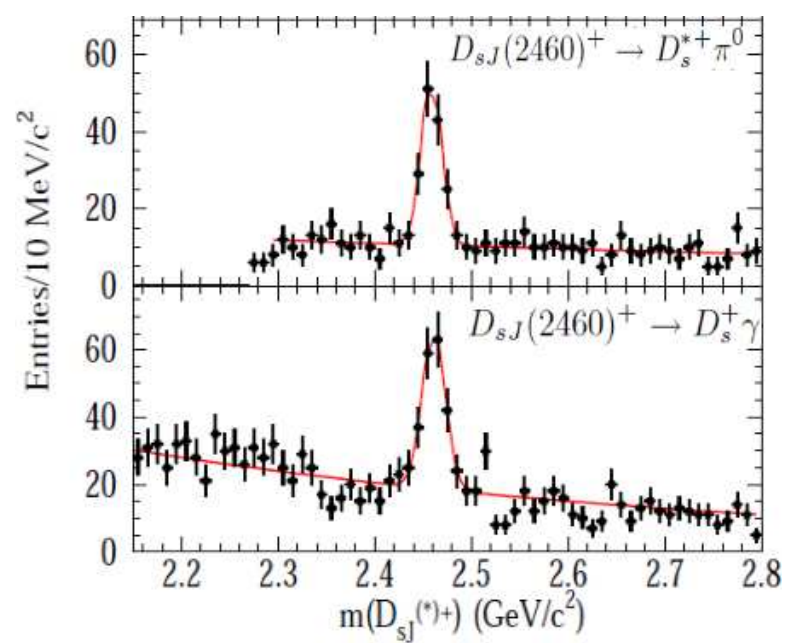

(b)

Figure 3: (a) $D_{S}^{+} \pi^{0}$ invariant mass system, in the inclusive analysis of $e^{+} e^{-} \rightarrow \bar{c} c$ at the energy near $10.6 \mathrm{GeV}$ (BaBar data) [3]: a clear peak at the mass $\mathrm{M}=2.371 \mathrm{GeV} / \mathrm{c}^{2}$ is observed. (b) Observation of $D_{s 1}(2460)^{+}$at BaBar in two different decay modes [4].

- a chiral symmetry breaking test;

- the study of mixing of $D_{S J}$ states with same spin and parity;

- study of the invariant mass system of $D_{s} D_{s J}^{*}$, and search for 4-quark states with strange content in the Charmonium field.

In this report we only discuss the first two items. The next section reports the status of the $\overline{\mathrm{P} A N D A}$ simulations.

\subsection{Strategy}

In PANDA, the framework used to simulate $\bar{p} p \rightarrow D_{s}^{-} D_{s 0}^{*}(2317)^{+}$events is PandaRoot [18]. It is based on Virtual Monte Carlo (VMC), that makes use of Geant 3 [19] for this specific analysis. A full simulation is performed, with a detailed magnetic B field map (constant $\mathrm{B}=2 \mathrm{~T}$ in the central part; $\mathrm{B}=2 \mathrm{~T} \cdot \mathrm{m}$ in the dipole area). Signal events are simulated using the Monte Carlo (MC) generator EvtGen [20]; background events are simulated using the Dual Parton Model (DPM) [21]. The reconstruction chain under study is: $\bar{p} p \rightarrow D_{s}^{-} D_{s 0}^{*}(2317)^{+}, D_{s}^{-} \rightarrow K^{+} K^{-} \pi^{-}$. In an exclusive reconstruction process, we would have: $D_{s 0}^{*}(2317)^{+} \rightarrow D_{s}^{+} \pi^{0}, \pi^{0} \rightarrow \gamma \gamma$. The model used to simulate $D_{s}$ events is the so called DS-DALITZ, which reproduces all internal structures of the $K \pi$ and $K K$ systems in the $D_{s}^{+} \rightarrow K^{+} K^{-} \pi^{+}$decay. The DS-DALITZ model is part of EvtGen, and it is based on the BaBar/CLEO data (see Fig. 4). 

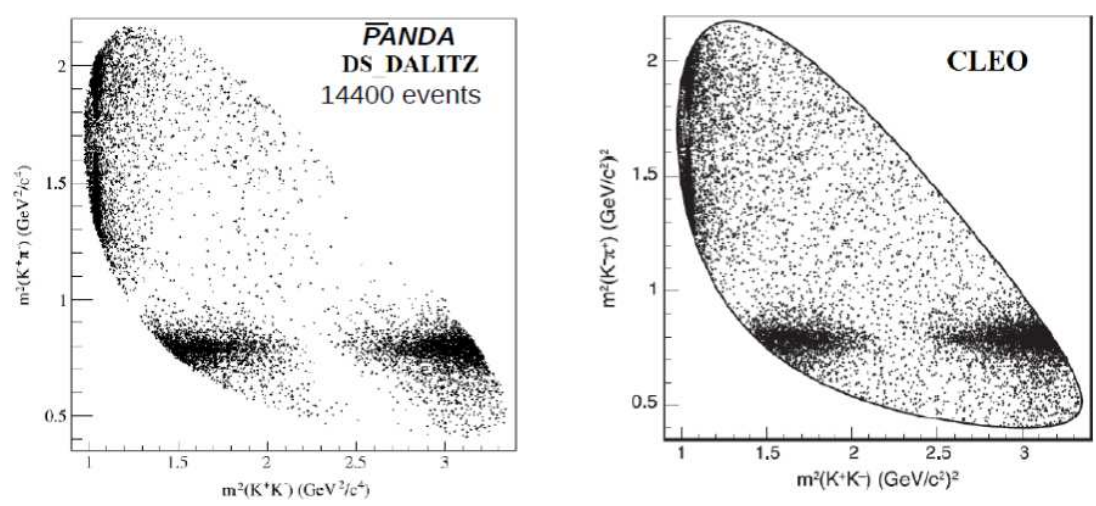

Figure 4: Simulation in Pandaroot for the reconstruction of $D_{s}^{+} \rightarrow K^{+} K^{-} \pi^{+}$in the process $\bar{p} p \rightarrow D_{s}^{+} D_{s}^{-}$: 14400 events have been generated using the DS-DALITZ model in EvtGen (left); Dalitz plot based on real data from the CLEO experiment for $D_{s}^{+} \rightarrow K^{+} K^{-} \pi^{+}$(right) [22].

At the production threshold of the $D_{s}^{-} D_{s 0}^{*}(2317)^{+}$system in $\bar{p} p$ annihilation, the antiproton beam is set to $p_{\text {beam }}=8.80235 \mathrm{GeV} / \mathrm{c}$, corresponding to an invariant mass of the system $\mathrm{M}_{\text {tot }}=$ $4.286 \mathrm{GeV} / \mathrm{c}^{2}$. Particle identification is used to separate kaons from pions. A cut on the photon momentum $\left(p_{\gamma}>50 \mathrm{MeV} / \mathrm{c}\right)$ and track momentum $\left(p_{\text {track }}>100 \mathrm{MeV} / \mathrm{c}\right)$ is applied at the level of an event pre-selection. This reduces the background due to very low momentum tracks. To improve the mass resolution and efficiency, an inclusive study in a single-tag mode is performed. This implies that we fully reconstruct the $D_{s}$ meson via the detected decay particles, and optimize a dedicated selection. We thus obtain the $D_{s 0}^{*}(2317)^{+}$as missing mass of the event $\bar{p} p \rightarrow D_{s}^{-} D_{s 0}^{*}(2317)^{+}$.

We perform two studies in parallel: an inclusive study including 3 different $D_{s}^{(*)}$ states, as reported in Fig. 5(a), and a specific simulation where only the excited $D_{s}$ state is the $D_{s 0}^{*}(2317)^{+}$ (see Fig. 5(b)). In both figures, only the pre-selection has been applied, and we select $\sim 30 \%$ of $D_{s}$ on a sample of 10000 generated events. A mass resolution of $\sim 16 \mathrm{MeV} / \mathrm{c}^{2}$ is obtained by a Gaussian fit. The background cross section is evaluated to be in the order of $\sim 2.2 \mathrm{mb}$, which has to be compared with a signal cross section of the order of a few nb. The rejection of the high combinatorial background is one of the main challenges of this analysis.

\subsection{A dedicated selection for $\bar{p} p \rightarrow D_{s}^{-} D_{s 0}^{*}(2317)^{+}$reaction}

Once the pre-selection is fixed, a dedicated selection is studied. It involves kinematic variables, such as the cosine of the angles among the $D_{s}$ daughters, the $p_{t, z}$ momentum resolution, the $D_{s}$ decay vertex position, and $\Delta E$. The variable $\Delta E$ is defined as the difference between the measured energy in the center of mass system, and its expected value. It can be parameterized by a double Gaussian function for signal events, centred around zero, and a polynomial function for background events.

Figure 6 shows the results of this preliminary selection. The $D_{s 0}^{*}(2317)^{+}$is evaluated as missing mass of the event; thus, the mass resolution of the $D_{s}^{-} D_{s 0}^{*}(2317)^{+}$system is expected to be 


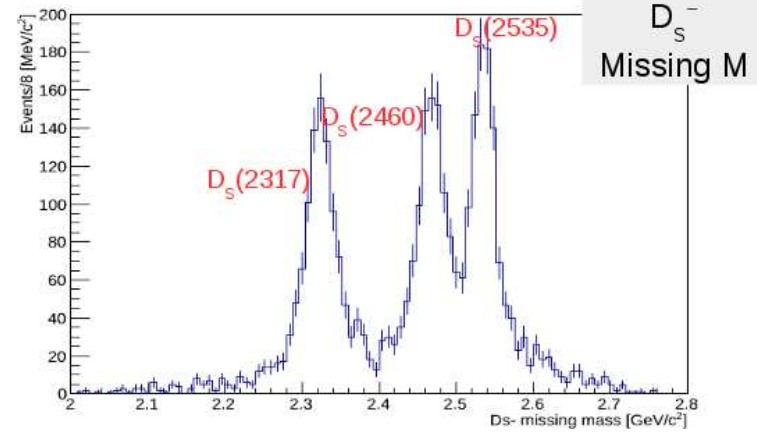

(a)

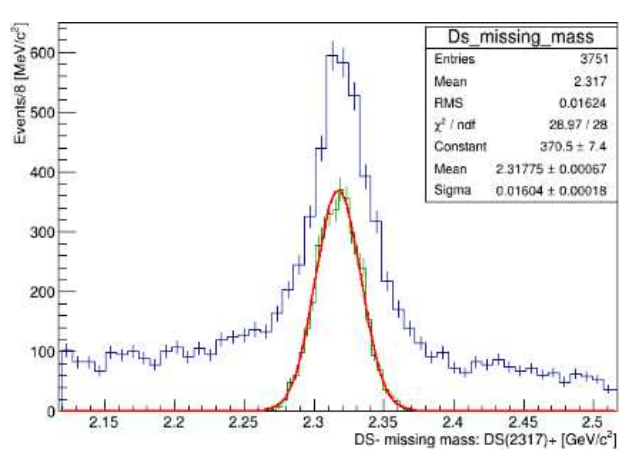

(b)

Figure 5: (a) Mass values of several $D_{S J}$, in an inclusive analysis of the channel $\bar{p} p \rightarrow D_{s}^{-} D_{s J}^{(*)+}$, as indicated. (b) Inclusive reconstruction of $D_{s 0}^{*}(2317)^{+}$in the process $\bar{p} p \rightarrow D_{s}^{-} D_{s 0}^{*}(2317)^{+}$. The blue curve represents signal + combinatorial background; the red curve fits the signal shape (green distribution). For this analysis, 10000 events have been simulated.

much more improved compared to that of the $D_{s}^{-}$and the $D_{s 0}^{*}(2317)^{+}$, shown in Fig. 6(a) and Fig. 6(b), respectively.

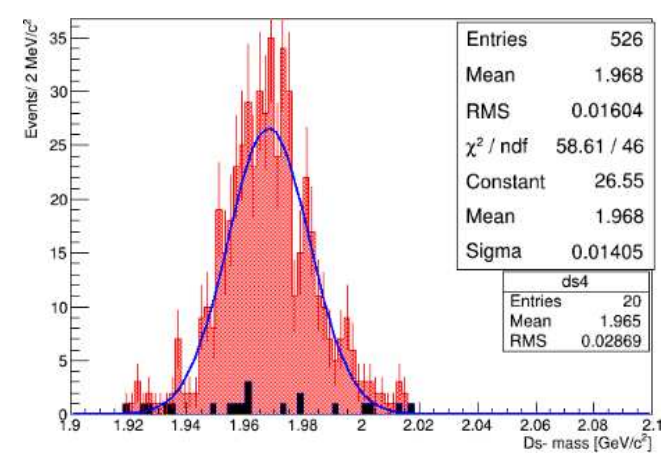

(a)

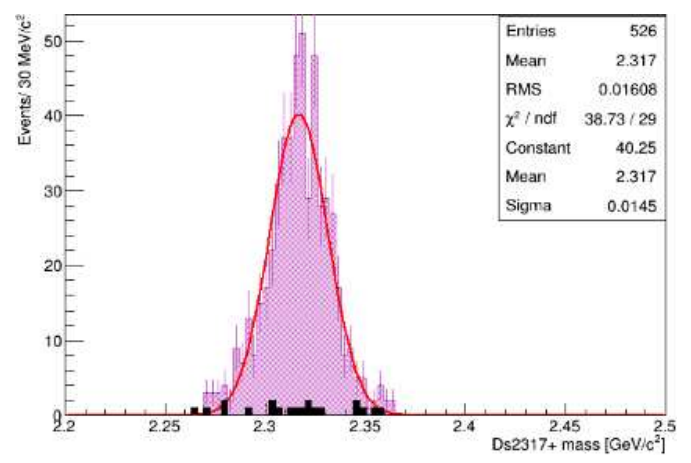

(b)

Figure 6: (a) The reconstructed $D_{s}$ mass refers to the $K^{+} K^{-} \pi^{-}$system. Comparison between signal (red) and background (black), after a dedicated selection. (b) The $D_{s 0}^{*}(2317)^{+}$mass refers to the missing mass of the event in the $\bar{p} p \rightarrow D_{s}^{-} D_{s 0}^{*}(2317)^{+}$process. Comparison between signal (violet) and background (black), after a dedicated selection. Under the hypothesis of a signal cross section equal to $20 \mathrm{nb}$, the background sample has to be scaled by a factor 60 .

We plan to scan the invariant mass system of $D_{s}^{-} D_{s 0}^{*}(2317)^{+}$in $100 \mathrm{keV} / \mathrm{c}^{2}$ steps, at 15 energyscan points. We need to collect many points as the $D_{s 0}^{*}(2317)^{+}$line shape is not known at all. We will extract the excitation function of the cross section as function of the energy; at the production theshold of the $D_{s}^{-} D_{s 0}^{*}(2317)^{+}$pair, the excitation function of the cross section depends on the 
mass and width of the resonant state. In Fig. 7 this curve is shown, for different input values of the width [24].

With the preliminary selection described in this report, we obtain a reconstruction efficiency

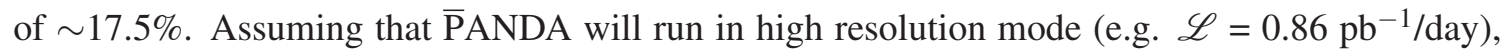
for which we tag the $D_{s}^{-} \rightarrow K^{+} K^{-} \pi^{-}\left(\mathrm{BR}\left(D_{s}^{-} \rightarrow K^{+} K^{-} \pi^{-}\right) \sim 6 \%\right)$, in the hypothesis to run 3000 events per scan point, we will need from 7 up to 11 days of data taking per scan point, on the signal cross section assumed to be in the range of [20;30] nb, as supported by Ref. [13]. In this situation, the ratio of signal $(\mathrm{S})$ over background $(\mathrm{B})$ events is $\mathrm{S} / \mathrm{B}=1 / 28$. Before appling this preliminary selection, $\mathrm{S} / \mathrm{B}=1 / 10^{6}$.

For comparison, in the first 3 years of data taking, the BaBar experiment recorded 1267 event yield. Therefore, we conclude that with $\bar{P}$ ANDA the reconstruction efficiency of the $D_{s 0}^{*}(2317)^{+}$ is significantly higher than at the $\mathrm{B}$ factories. The performance of the proposed inclusive analysis, based on PandaRoot MC simulations, looks therefore promising.

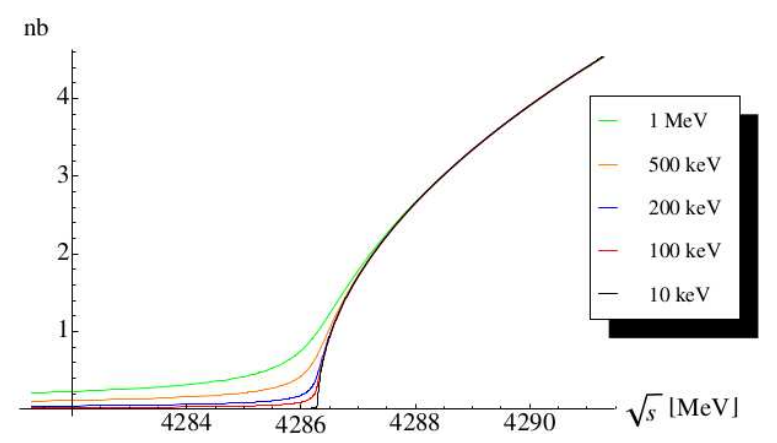

Figure 7: Excitation function of the cross section of the process $\bar{p} p \rightarrow D_{s}^{-} D_{s 0}^{*}(2317)^{+}$, as function of the energy in the center of mass system of $D_{s}^{-} D_{s 0}^{*}(2317)^{+}$. The plot is taken from Ref. [24].

\section{Summary}

Charm physics is a field of high interest, in which several questions are still unsolved. Among these, the measurement of the width of the narrow $D_{s}$ states below the DK threshold is important to fully understand the $c \bar{s}$ spectrum. PANDA offers a unique opportunity to perform this measurement, and the results of simulations with PandaRoot are very promising. This measurement cannot be performed in the early stage of PANDA data taking. The cross section of the process $\bar{p} p \rightarrow D_{s}^{-} D_{s 0}^{*}(2317)^{+}$could be estimated to be in the range [20;30] nb (as explained in Ref. [13]), thus in the worse scenario, corresponding to a cross section of $20 \mathrm{nb}$, we would need of about 165 days to perform the full scan of the $D_{s}^{-} D_{s 0}^{*}(2317)^{+}$system. The results that we aim for, will reach a level of precision never achieved before. Therefore, we consider this analysis a highlight of the PANDA physics program. The optimization of the selection, and the numbers quoted in this report, are work in progress. We plan to complete the first full simulation campaign for the analysis of $\bar{p} p \rightarrow D_{s}^{-} D_{s 0}^{*}(2317)^{+}$by the summer 2015. 


\section{References}

[1] S. Godfrey and N. Isgur, Phys. Rev. D 32, 189 (1985).

[2] M. di Pierro and E. Eichten, Phys. Rev. D 64, 114004 (2001).

[3] The BaBar Coll., Phys. Rev. Lett. 90, 242001 (2003).

[4] The BaBar Coll., Phys, Rev. Lett. 93, 181801 (2004).

[5] L. Liu et all., Phys. Rev. D 87, 014508 (2013).

[6] H. Y. cheng et all.,Phys. Lett. B 566, 193 (2003).

[7] S. Godfrey, Phys. Lett. B 568, 254 (2003).

[8] J. Beringer et al. (PDG Collaboration), Phys. Rev. D 86, 010001 (2012).

[9] M. Cleven et al., Eur. Phys. J. A, 50 (2014).

[10] The PANDA Coll., arXiv:0903.3905 [hep-ex].

[11] E. Braaten and P. Artoisenet, Phys. Rv. D 79, 114005 (2009).

[12] A. Khodjamirian et al., Eur. Phys. J. A, 31 (2012).

[13] J. Heidenbauer, G. Krein, Phys. Rev. D 89, 114003 (2014).

[14] D. Ebert et al., Eur. Phys. J. C 66, 197 (2010).

[15] G. S. Bali, Phys. Rev. D 68, 071501 (2003).

[16] C. B. Lang et al., Phys. Rev. D. 90, 034510 (2014).

[17] The E760 Coll., Phys. Rev. D 47, 772 (1993).

[18] S. Spataro, Journal of Physics: Conference Series 396, 022048 (2012).

[19] R. Brun et al., CERN-DD-EE-84-1 (1987).

[20] D. J. Lange, Nucl. Instr. Methods A 462, 152 (2001).

[21] A. Cappella et al., Physics Report 236, 225 (1994).

[22] The CLEO Coll., Phys. Rev. D 79, 072008 (2009).

[23] The LHCb Coll., Phys. Rev. Lett. 113, 162001 (2014).

[24] http://www-panda.gsi.de/db/thesesDB/. 